\title{
Developmental Changes in Cognitive Bias
}

\author{
Akiko Suzuki' ${ }^{1}$, Akiko Megumi1 ${ }^{1}$, Akira Yasumura² \\ ${ }^{1}$ Graduate School of Social and Cultural Sciences, Kumamoto University, Kumamoto, Japan \\ ${ }^{2}$ Graduate School of Humanities and Social Sciences, Kumamoto University, Kumamoto, Japan \\ Email: suzukiakiko219@gmail.com
}

How to cite this paper: Suzuki, A., Megumi, A., \& Yasumura, A. (2021). Developmental Changes in Cognitive Bias. Psychology, 12, 293-303.

https://doi.org/10.4236/psych.2021.122019

Received: January 18, 2021

Accepted: February 23, 2021

Published: February 26, 2021

Copyright $\odot 2021$ by author(s) and Scientific Research Publishing Inc. This work is licensed under the Creative Commons Attribution International License (CC BY 4.0).

http://creativecommons.org/licenses/by/4.0/

\begin{abstract}
While many studies have investigated incorrect evaluations of cognitive bias type and how age-related decisions are influenced by it, few have compared this phenomenon across different age groups (i.e., adults and children). In this study, we examined developmental changes by comparing the number of correct answers given by children and that given by adults when performing different types of cognitive bias tasks. The participant sample comprised 36 children ( 21 boys and 15 girls with an average age of 12.3 years) and 26 adults (15 men and 11 women with an average age of 27.3 years). The cognitive bias task created by previous study was modified and then implemented to better understand the performance of the children. This study identified four types of developmental changes in cognitive bias. Consequently, it was suggested that in terms of cognitive reflection, conjunction bias and confirmation bias improved performance, and framing did not change for either children or adults. It was also suggested that the confirmation bias tasks' lack of consideration for the subcategory of ADHD was associated with performance among both the children and the adults. Results show that some cognitive biases may reveal developmental changes, depending on the category. As such, we were able to consider the factors involved in human decision-making and obtain a perspective on developmental changes to improve future decision-making.
\end{abstract}

\section{Keywords}

Cognitive Biases, Developmental Changes, Inattention

\section{Introduction}

According to Kahneman, Slovic, and Tversky (1982), people use the empirical rule of heuristics when making judgments. Heuristics are intuitive and are considered a quick and easy means of making accurate and adaptive decisions. Heuristics are used because human beings have limited ability to memorize informa- 
tion and calculate probabilities.

However, humans struggle to understand the world objectively and calmly. Social beliefs in everyday situations may compel unfounded views. Such views are collectively referenced as cognitive bias. Different reported types exist, depending on the context in which the bias occurs. Various biases occur regardless of age (Takahashi \& Omori, 2011).

Amid the occurrence of various cognitive biases, human beings must possess mature understanding (based on meaning rather than literal interpretations) to enable improved decision-making. This mature understanding is not native; a mature understanding is cultivated over time through development processes.

Children and adolescents tend to lack a mature understanding of situations and consequently, make immature decisions. Adults begin making decisions based on the meaning of the information rather than the details (Reyna \& Brainerd, 2011). Younger people tend to make evaluations based on the risk of verbatim information rather than the qualitative "gist;" as such, they tend to have poor judgment (Reyna \& Farley, 2006). Conversely, adults tend to make judgments that involve calculated risks, through which they may expose themselves to danger based on a greater purpose rather than statistical data. In general, however, this group tends to be risk-averse.

Wood, Busemeyer, Koling, Cox, and Davis (2005) show that older people are more likely to pay attention to events that are expected to be beneficial and make choices that will bring about short-term benefits. As we age, many of our cognitive resources are allocated to our emotions; hence, older people make more automatic decisions (Carstensen, Mikels, \& Mather, 2006). Thus, elderly people tend to be targeted for crimes of opportunity, such as phone fraud (Nagamine, Hara, \& Nobuhara, 2009).

Human decision-making is not static throughout life; rather, it undergoes developmental changes that are affected by experience and aging. However, decision-making studies often target college students through surveys or experiments. Few studies have compared the decision-making abilities of different age groups (Bruine de Bruin, 2012; Fischhoff, 2008; Peters \& Bruine de Bruin, 2012; Strough, McKarns, \& Schlossnagle, 2011). Additionally, few studies have examined the tendency for cognitive bias among children, and among the various cognitive biases, it is unclear which kind of bias is affected by developmental changes.

By clarifying the developmental changes in cognitive bias, we believe clues to enable improved decision-making will be revealed, as each developmental stage will be isolated. Accordingly, this study examined developmental changes in cognitive bias among children and adults.

\section{Method}

\subsection{Research Participants}

The participants included 36 children enrolled in a regular class ( 21 boys and 15 girls with an average age of 12.3 years) and 26 adults ( 15 men and 11 women 
with an average age of 27.3 years). We described the experiment for the participants and their guardians. The children, guardians, and adults provided their informed consent to participate in the study.

\subsubsection{Autism-Spectrum Quotient (AQ)}

We asked parents to respond to a questionnaire about the participating children, and the participating adults responded to the questionnaire on their own behalf. The AQ is an international screening test that measures an individual's autism tendency and identifies autism spectrum disorders; it has five subscales comprising social skills, attention switching, attention to detail, communication, and imagination.

\subsubsection{Child ADHD Rating Scale (Conners 3)}

We asked the participating children's teachers to respond to a questionnaire about them. Conners 3 is widely used to assess children with ADHD-related problems and has five subscales, which comprise carelessness, hyperactivity/aggression, learning problems/executive function, defiance challenge/aggression, and friendship.

\subsubsection{Adult ADHD Rating Scale (CAARS)}

We asked the participating adults to complete a questionnaire. The CAARS is a questionnaire that can measure ADHD symptoms in adults and has four subscales, which comprise inattention/memory, hyperactivity/restlessness, impulsivity/emotional lability, and self-concept.

\subsubsection{Raven Color Matrix Test (Raven's Colored Progressive Matrices [RCPM])}

The participants performed a general intelligence test based on the RCPM (gfactor). The RCPM is a simple intelligence test developed to measure Spearman's general g-factor. Among the study participants, we excluded one child who was diagnosed using ASD tests and for whom the RCPM correct answer rate was less than $50 \%$.

This study was approved by the Ethics Review Committee, Faculty of Humanities and Social Sciences, at Kumamoto University (No. 45).

\subsubsection{Creating Cognitive Bias Tasks for Children}

Suzuki, Megumi, and Yasumura (2020) used measures for adults from previous studies. Issues stemming from cognitive bias were extracted using four categories and modified to fit the contents and expressions that precisely align with the structure of Japanese elementary schools. In this study, to perform versatile tasks and conduct experiments, we modified the proper nouns and personal names that appear in the task to reflect general nouns instead.

\subsection{Creation of the Cognitive Bias Task}

We used measures for adults from previous studies on cognitive bias issues extracted using four categories and modified them to fit the contents and expres- 
sions to directly leverage the structure of the issues confronting Japanese elementary schools.

<Example of conjunction bias task created for elementary school students>

Taro is a shy 12-year-old boy. He excels in science. His hobby is collecting stones. On his way home from school, he searches for rare stones. He arrives home and has more than 10 stones lined up. Choose the description that best fits Taro.

1) Taro is an environmental committee member.

2) Taro is a nerdy environmental committee member.

3) Taro is an unmotivated committee member.

$<$ Example of cognitive reflection tasks created for elementary school students>

Fallen leaves float in a pool. The area covered by fallen leaves continues to increase every two days. Leaves fall for 48 days until they completely cover the pool. How many days does it take to cover half of the pool? Choose one of the following:

1) 12 days

2) 24 days

3) 47 days

In this way, elementary school students tend to create a provisional problem with content that can be fully understood. Further, a preliminary experiment was conducted to target 36 children in sixth grade at an elementary school. We calculated the correct rate of the task and applied a curve based on its level of difficulty. This was carried out approximately two months after the second preliminary experiment was carried out to target 36 children in sixth grade at the same elementary school. The correct answer rate was calculated for the second time. The standard deviation of $\pm 2 \mathrm{SD}$ or more was omitted, and the expression was adjusted for linguistic ease.

\subsection{Experimental Procedure}

The pre-recorded stimuli were automatically presented on a laptop using PsychoPy. The recording was played, and the participant reaction was obtained by pressing the button. The participants were taught to perform this protocol as accurately and quickly as possible. Before completing the actual task, a practice trial with a lower cognitive load was performed. We attempted a total of 21 questions, three for each of the four categories of biases and nine questions for the dummy task. After replaying the protocol, we requested an answer by pressing the "OK" button. After obtaining the correct answer, the next question appeared (no feedback was provided to the participant). The experiment was conducted in a quiet environment, such as an empty classroom or laboratory.

The Ethics Committee of the Graduate School of Social and Cultural Sciences of Kumamoto University approved the protocol of the present study (approval unmer: 45). 


\subsection{Statistical Analysis}

In addition to the number of correct answers for each of the four types of cognitive bias tasks, the total number of correct answers for bias tasks, the number of correct answers for non-bias tasks, and the total score for the child and adult groups were compared at a significance level of 5\%. A given student's t-test or Welch's t-test was performed after confirming whether homoscedasticity was present according to the $\mathrm{F}$ test.

For the children, we calculated the median scores of Conners (for each differentiation of five subscales) and divided the high and low groups of scores. For the adults, we calculated the median scores from the CAARS (for each differentiation of five subscales) and divided the high and low groups of scores. At a significance level of 5\%, we compared the groups' total scores from the high and low confirmation bias task. Again, a given student's t-test or Welch's t-test was performed after confirming whether homoscedasticity was present according to the $\mathrm{F}$ test.

The effects of the development (2; pediatric/Adult) and task category (4; Cognitive reflection/conjunction bias/framing/confirmation bias) were investigated via a two-way analysis of variance (ANOVA).

\section{Results}

A t-test revealed the low- and high-scoring groups for the Conners 3 learning problem/executive function. The mean scores from the confirmation bias task were 1.78 in the low group and 1.11 in the high group. A significant difference was observed $(\mathrm{t}[34]=2.249, p=.031)$, as shown in Figure 1 .

The results of the t-test performed on the low- and high-scoring groups of the Conners 3 inattention reveal mean scores for the confirmation bias task: the low group scored 1.68 and the high group scored 1.18. A significant trend was observed $(\mathrm{t}[34]=1.891, p=.067)$, as shown in Figure 2 .

In contrast to the scores from the conjunction bias problems, the results of the t-test performed on the low- and high-scoring groups of the Conners 3 inattention revealed scores of 0.89 for the low group and 1.24 for the high group. No significant difference was observed $(\mathrm{t}[34]=-1.244, p=.222)$.

In contrast to the mean scores from the confirmation bias task, the results of the t-test performed on the low- and high-scoring groups of the CAARS revealed scores of 2.69 for the low group and 2.21 for the high group. A significant difference was observed ( $\mathrm{t}[25]=2.325, p=.029$ ), as shown in Figure 3 .

Unlike the scores for the Cognitive reflection task, the results of the t-test performed on the low- and high-scoring groups of the CAARS hyperactivity/restlessness revealed scores of 2.70 for the low group and 2.35 for the high group. No significant difference was observed $(\mathrm{t}[25]=1.541, p=.136)$. See Figure 4.

We performed a two-factor ANOVA (Development [2; Pediatric/Adult] $\times$ Task Category [4; Cognitive reflection/framing/conjunction bias/confirmation bias]) for the developmental and cognitive bias tasks. Consequently, main effects were observed for the correct answer rates for the developmental and task categories (development: $\mathrm{F}[1,61]=41.157, p<.001$; task category: $\mathrm{F}[3,183]=$ 
$10.184, p<.001)$. The development and task categories exhibited significant interactions $(\mathrm{F}[3,183]=7.066, p<.001)$. An analysis of the interaction revealed a simple effect from development in each category. The adults had higher values in cognitive reflection, conjunction bias, and confirmation bias than the children (cognitive reflection: $\mathrm{F}[1,244]=39.904, p<.001$; framing: $\mathrm{F}[1,244]=0.230, p$ $=.632$; conjunction bias: $\mathrm{F}[1,244]=4.745, p<.05$; confirmation bias: $\mathrm{F}[1,244]$ $=22.137, p<.001)$. See Figure 5 .

Differences between the adults and the children were observed in the main effects of the task categories (adults: $\mathrm{F}[3,183]=9.269, p<.001$; children: $\mathrm{F}[3$, $183]=7.982, p<.001)$. Following multiple comparisons, adults scored higher on cognitive reflection than on conjunction bias $(p<.05)$. Additionally, their confirmation bias scores were higher than those for conjunction bias $(p<.05)$. The framing scores were higher than those for conjunction bias $(p<.05)$. The other combinations did not differ significantly $(p>.05)$. Among the children, the framing scores were higher than those for conjunction bias $(p<.05)$. The framing scores were higher than those for cognitive reflection $(p<.05)$. Furthermore, the framing scores were higher than those for confirmation bias ( $p$ $<.05)$. The other combinations did not exhibit significant differences $(p>.05)$.

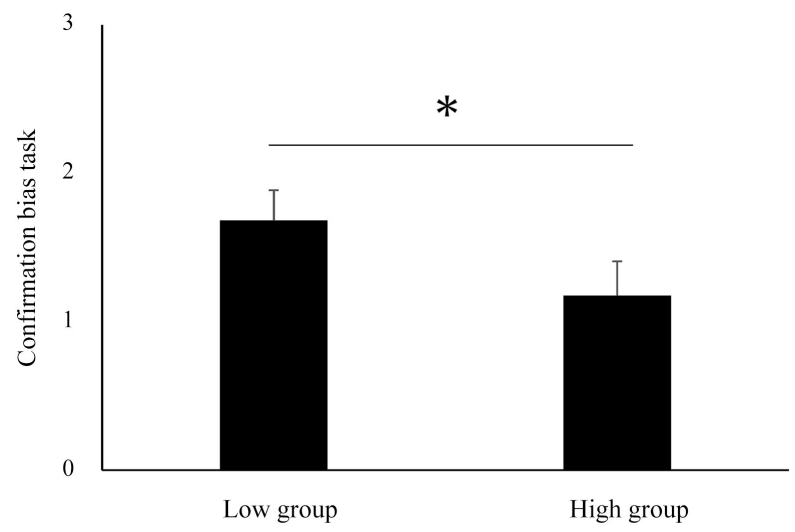

Figure 1. Comparison of the number of correct answers in learning/executive function (Child). Error bars indicate standard errors. ${ }^{*}, p<.05$.

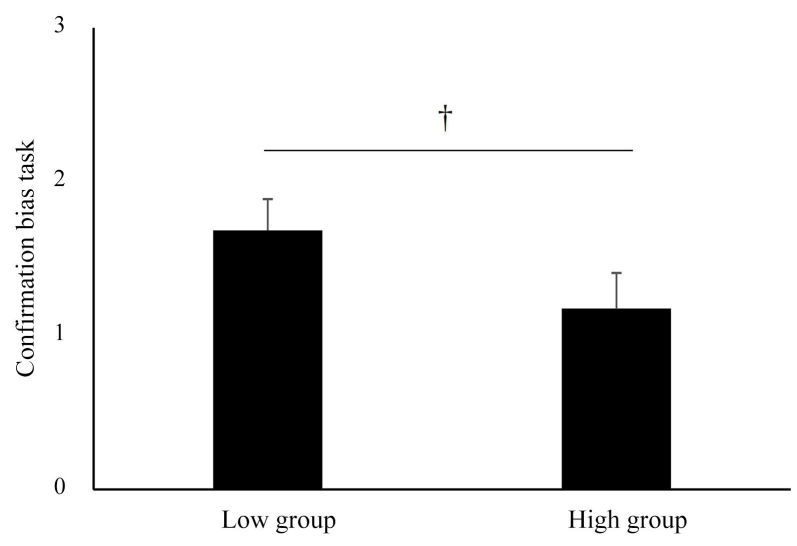

Figure 2. Comparison of the number of correct answers for inattention (Child). Error bars indicate standard errors. $\dagger, p<.10$. 


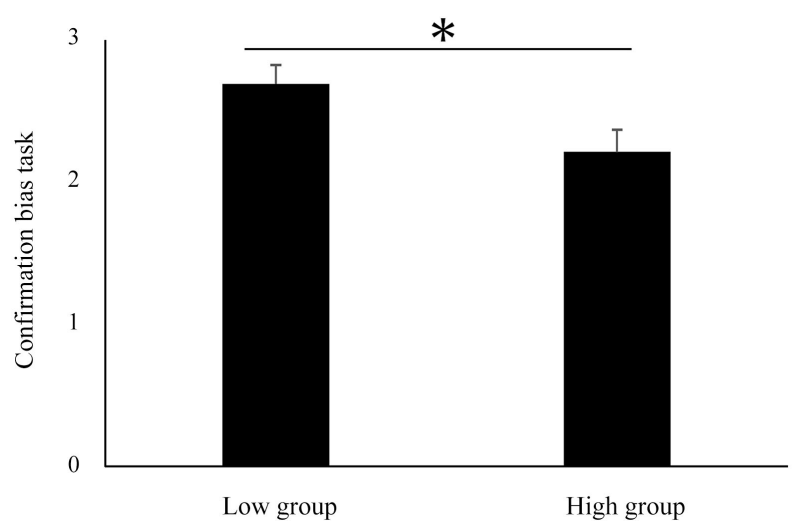

Figure 3. Comparison of the number of correct answers in inattention (Adult). Error bars indicate standard errors. ${ }^{\star}, p<.05$.

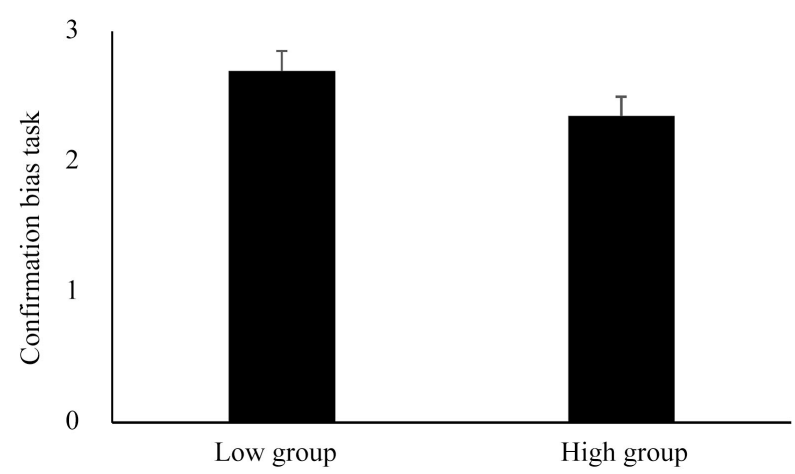

Figure 4. Comparison of the number of correct answers in hyperactivity (Adult).

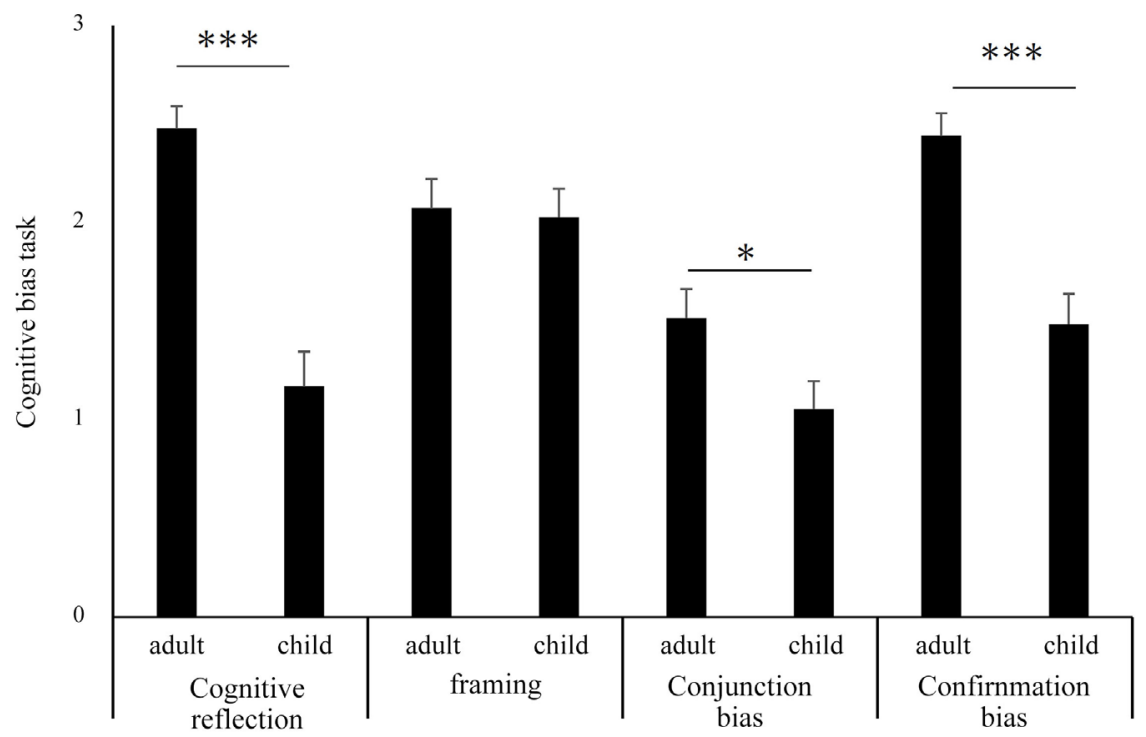

Figure 5. Comparison of the number of correct answers by task. Error bars indicate standard errors. ${ }^{*}, p<.05,{ }^{* * *}, p<.001$.

\section{Discussion}

This study's principal aim was to clarify how the development of four types of 
cognitive bias (conjunction bias, framing, confirmation bias, and cognitive reflection) influence human decision-making from childhood to adulthood. The results of investigating developmental changes in the four types of cognitive bias in the confirmation bias and cognitive reflection tasks showed that adults gave a higher number of correct answers than children.

In Japan's current public education curriculum, probability is not taught in elementary school mathematics and students learn it for the first time in their second year of junior high school. However, children make probability judgements in their lives and form an unsystematic probability concept as a dynamic notion (Matsuura, 2006). In contrast, adults have learned numeracy through their education and life experiences. Studies on probabilities state that education levels influence probability theory (Hertwig, Zangerl, Biedert, \& Margraf, 2008).

In developmental research on schools, ranging from second-grade elementary schools to third-grade junior high, Toplak, West, and Stanovich (2013) showed that the ratio bias gradually decreased as the grade increased. In the case of the ratio bias, it is necessary to activate the inhibitory function to avoid mistakes, such as ignoring the denominator. However, age improves the ability to suppress the dominant reaction; it has been concluded that ratio bias is reduced and judgment improves (Reyna, 2004; Reyna \& Mills, 2007). Inhibitory function is one of the three components of executive function (Miyake, Friedman, Emerson, Witzki, \& Howerter, 2000) and has been shown to mature over a long period until adolescence (Gogtay et al., 2004). Given the development of executive functions, adults have more suppressive responses than children.

The number of correct answers in cognitive reflection, framing, and confirmation bias among adults was higher than those for conjunction bias. Additionally, the conjunction bias showed that adults had a higher number of correct answers than children. Conjunction bias is based on the concept of representativeness (Tversky \& Kahneman, 1983). In conjunction bias issues, misunderstanding in a task occurs as a conjunction fallacy (Hertwig \& Gigerenzer, 1999). In tasks that involve an estimate of probability, misunderstandings about the meaning of problems and choices cause conjunctive mistakes. Meanwhile, Ninomiya and Fujiki (2018) found that the difference in memory representation, which predicts the presence or absence of a conjunction fallacy in the "Linda" task, is not owing to the misunderstanding of the task. Differences in memory representation occur depending on heuristic judgment. Suzuki et al. (2020) created conjunction tasks with a problem structure like that of the "Linda" conjunction bias task. Adults solve the task using a conjunctive rather than a probability judgment.

Among the children, the correct answer rate for the framing task was higher than that of the other three task categories. Regarding the framing effect, Schlottmann and Tring (2005) used a game similar to roulette to evaluate the risk of gaining or losing jellybeans for children aged $6-7$ and $8-10$ years old. Consequently, in their efforts to avoid the risk of the acquisition frame and their risk orientation in the loss frame, adults show a reverse preference that is similar to 
that of the children. These results show that the development of the framing effect begins in early childhood, thus supporting Levin and Hart's (2003) theory (Sasaki, 2010). The results further reveal that the scores of participants aged 11 or 12 years old for the framing task were high and similar to those of the adults.

We compared the number of correct answers in the confirmation bias task between the high- and low-scoring inattentive groups who were evaluated using the ADHD subscale. In both the adults and the children, the group with a high level of inattention revealed lower scores than the low group. Among the children, the group with a higher learning or executive function performance performed more poorly than the group with less severe learning/executive function.

Executive function is considered to contribute to retaining information regarding a trigger problem, switching attention to various aspects of the problem, and preventing the problem from being affected by prominent or incorrect information (i.e., a snag problem) (Blair \& Razza, 2007).

It is probable that the group that scored high in carelessness (meaning they struggled to suppress unnecessary thoughts and focus on the task) was affected by the prominent and incorrect information contained in the task.

\section{Summary}

This study identified four types of developmental changes in cognitive bias. Consequently, it was suggested that conjunction bias and confirmation bias improved cognitive reflection performance, and framing did not change for either the children or the adults. It was also suggested that the confirmation bias tasks' lack of consideration for the subcategory of ADHD was associated with the performance of both the children and the adults. Based on this result, we were able to consider the factors involved in human decision-making and obtain a perspective on developmental changes that may prompt improved decision-making.

\section{Limitations of This Study}

It is not possible to observe developmental changes throughout life. As the participants in this study were children and adults, this study excluded elderly individuals. It is necessary to clarify which type of cognitive bias changes and how it adjusts from adulthood to mature adulthood.

\section{Future Outlook}

In the future, we would like to collect data on elderly individuals to investigate developmental changes in cognitive bias from adulthood to the most advanced stage of mature adulthood.

\section{Acknowledgements}

This research was supported by the Scientific Research Foundation (19K14300, Representative: Akira Yasumura). We would also like to thank everyone in the Psychology Laboratory, Faculty of Letters, Kumamoto University for their coop- 
eration in conducting this research.

\section{Conflicts of Interest}

The authors declare no conflicts of interest regarding the publication of this paper.

\section{References}

Blair, C., \& Razza, R. (2007). Relating Effortful Control of Executive Function and Understanding of False Belief in Emerging Math and Literacy Ability in Kindergarten. Child Development, 78, 647-663. https://doi.org/10.1111/j.1467-8624.2007.01019.x

Bruine de Bruin, W. (2012). Judgment and Decision Making in Adolescents. In M. K. Dhami, A. Schlottemann, \& M. Waldmann (Eds.), Judgment \& Decision Making as a Skill: Learning, Development, \& Evolution (pp. 85-112). New York: Cambridge University Press. https://doi.org/10.1017/CBO9781139015684.008

Carstensen, L. L., Mikels, J. A., \& Mather, M. (2006). Aging and the Intersection of Cognition, Motivation, and Emotion. In J. Birren, \& K. W. Schaie (Eds.), Handbook of the Psychology of Aging (pp. 343-362). New York: Academic Press. https://doi.org/10.1016/B9-78-012101-2/64950-0185

Fischhoff, B. (2008). Assessing Adolescent Decision-Making Competence. Development Review, 28, 12-28. https://doi.org/10.1016/j.dr.2007.08.001

Gogtay, N., Giedd, J. N., Lusk, L., Hayashi, K. M., Greenstein, D., Vaituzis, A. C., \& Toga, A. W. (2004). Dynamic Mapping of Human Cortical Development during Childhood through Early Adulthood. Proceedings of the National Academy of Science of the United States of America, 101, 8174-8179. https://doi.org/10.1073/pnas.0402680101

Hertwig, R. N., \& Gigerenzer, G. (1999). The “Conjunction Fallacy” Revisited: How Intelligent Inferences Look Like Reasoning Errors. Journal of Behavioral Decision Making, 12, 275-305.

https://doi.org/10.1002/(SICI)1099-0771(199912)12:4<275::AID-BDM323>3.0.CO;2-M

Hertwig, R. N., Zangerl, M. A., Biedert, E., \& Margraf, J. (2008). The Public's Probabilistic Numeracy: How Tasks, Education and Exposure to Games of Chance Shape It. Journal of Behavioral Decision Making, 21, 457-470. https://doi.org/10.1002/bdm.611

Kahneman, D., Slovic, P., \& Tversky, A. (1982). Judgment under Uncertainty: Heuristics and Biases. Cambridge: Cambridge University Press. https://doi.org/10.1017/CBO9780511809477

Levin, I. P., \& Hart, S. S. (2003). Risk Preferences in Young Children: Early Evidence of Individual Differences in Reaction to Potential Gains and Losses. Journal of Behavioral Decision Making, 16, 397-414. https://doi.org/10.1002/bdm.453

Matsuura, T. (2006). Longitudinal and Cross-Sectional Study on the Actual State of Probability Judgment of Children. Japan Academic Society of Mathematics Education, 12, 141151.

Miyake, A., Friedman, N. P., Emerson, M. J., Witzki, A. H., \& Howerter, S. (2000). The Unity and Diversity of Executive Functions and their Contributions to Complex "Frontal Lobe” Tasks: A Latent Variable Analysis. Cognitive Psychology, 41, 49-100. https://doi.org/10.1006/cogp.1999.0734

Nagamine, M., Hara, S., \& Nobuhara, Y. (2009). Bank Transfer Fraud: A Neuroscientific Approach. Sociotechnica, 6, 177-186. (In Japanese) https://doi.org/10.3392/sociotechnica.6.177

Ninomiya, Y., \& Fujiki, D. (2018). Relationship between Comprehension Process of Con- 
junction Fallacy Task and Judgement Depending on Representation Heuristic. Cognitive Studies, 25, 421-434.

Peters, E. M., \& Bruine de Bruin, W. (2012). Aging and Decision Skills. In M. K. Dhami, A. Schlottmann, \& M. Waldmann (Eds.), Judgment and Decision Making as a Skill: Learning, Development, and Evolution (pp. 113-140). New York: Cambridge University Press. https://doi.org/10.1017/CBO9781139015684.009

Reyna, V. F. (2004). How People Make Decisions That Involve Risk: A Dual Process Approach. Current Directions in Psychological Science, 13, 60-66. https://doi.org/10.1111/j.0963-7214.2004.00275.x

Reyna, V. F., \& Brainerd, C. J. (2011). Dual Process in Decision Making and Developmental Neuroscience: A Fuzzy-Trace Model. Developmental Review, 31, 180-206. https://doi.org/10.1016/j.dr.2011.07.004

Reyna, V. F., \& Farley, F. (2006). Risk and Rationality in Adolescent Decision Making: Implications for Theory, Practice, and Public Policy. Psychological Science in the Public Interest, 7, 1-44. https://doi.org/10.1111/j.1529-1006.2006.00026.x

Reyna, V. F., \& Mills, B. A. (2007). Interference Processes in Fuzzy-Trace Theory: Aging, Alzheimer's Disease, and Development. In C. MacLeod, \& D. Gorfein (Eds.), Inhibition in Cognition (pp. 185-210). Washington DC: APA Press.

https://doi.org/10.1037/11587-010

Sasaki, H. (2010). Three Types of Decision-Making Framing Effects from the Perspective of Infant Development and Childcare. Niigata Chuo University Hyosung Journal, 60, 55-72.

Schlottmann, A., \& Tring, J. (2005). How Children Reason about Gains and Losses: Framing Effects in Judgment and Choice. Swiss Journal of Psychology, 64, 153-171. https://doi.org/10.1024/1421-0185.64.3.153

Strough, J., McKarns, T. E., \& Schlossnagle, L. (2011). Decision-Making Heuristics and Biases across the Life Span. Annals of the New York Academy of Science, 1235, 57-74. https://doi.org/10.1111/j.1749-6632.2011.06208.x

Suzuki, A., Megumi, A. \& Yasumura, A. (2020). Relationships between Cognitive Bias and Autistic Traits in Children. Kumamoto University Studies in Social and Cultural Sciences, 18, 65-74.

Takahashi, H., \& Omori, T. (2011). The Role of Social Belief Effect in Social Interaction and Its Neural Mechanism. Cognitive Studies, 18, 138-157.

Toplak, M. E., West, R. F., \& Stanovich, K. E. (2013). Rational Thinking and Cognitive Sophistication: Development, Cognitive Abilities, and Thinking Dispositions. Developmental Psychology, 50, 1037-1048. https://doi.org/10.1037/a0034910

Tversky, A., \& Kahneman, D. (1983). Extensional versus Intuitive Reasoning: The Conjunction Fallacy in Probability Judgment. Psychological Review, 90, 293-315. https://doi.org/10.1037/0033-295X.90.4.293

Wood, S., Busemeyer, J., Koling, A., Cox, C. R., \& Davis, H. (2005). Older Adults as Adaptive Decision Makers: Evidence from the Iowa Gaming Task. Psychology and Aging, 20, 220-225. https://doi.org/10.1037/0882-7974.20.2.220 